\title{
Possibilidades educacionais para tecnologia podcast
}

\author{
Caique Ferreira $^{1}$, João Marcos Soares Anjos ${ }^{1}$, João Vinícius Ottoni ${ }^{1}$, \\ Milton P. de Almeida Castro ${ }^{1}$, Valguima Odakura ${ }^{1}$, Carla Adriana Barvinski ${ }^{1}$, \\ Rodrigo P. da Silva Sacchi ${ }^{1}$ \\ ${ }^{1}$ Faculdade de Ciências Exatas e Tecnologia (FACET) \\ Universidade Federal da Grande Dourados (UFGD) \\ Dourados-MS-Brasil \\ \{caiquef.ms, joaomarcoscomp, ottonijehad, castro.milton07, \\ valguima.odakura, carlabarvinski, rodrigosacchi.ufgd\}@gmail.com
}

\begin{abstract}
Podcasts are audio or video files distributed over the Internet, representing a mobile technology that has gained the audience of young people. In this context, the use of podcasts in education has been explored as a further way to mediate the learning at any time and place. In this article is proposed the development of an educational serie of podcasts for use by undergraduate students in Computer. After listening to the episodes, most students evaluated the podcasts as useful tools for learning of the proposed content.
\end{abstract}

Resumo. Podcasts são arquivos de áudio ou vídeo distribuídos pela Internet, que representam uma tecnologia móvel recente que tem ganhado a audiência dos jovens. Nesse contexto, a utilização de podcasts na educação vem sendo explorada como mais uma forma de mediar a aprendizagem a qualquer tempo e lugar. Neste artigo, propõe-se o desenvolvimento de uma série educacional de podcasts para ser utilizada por estudantes de graduação em Computação. Após ouvir a série, a maioria dos estudantes avaliou os podcasts como instrumentos úteis para aprendizagem do conteúdo proposto.

\section{Introdução}

O avanço na tecnologia de comunicação e redes sem fio, permitiu que tivéssemos dispositivos móveis, como smartphones e tablets, disponíveis a um menor custo. Os dispositivos atuais incorporaram a rede sem fio, email, tocadores de música, gravação de áudio e vídeo, entre outros. Esse avanço tecnológico impactou a sociedade como um todo, tendo também seu alcance na educação. Nesse contexto, os dispositivos móveis utilizados na educação culminaram na chamada aprendizagem móvel, do inglês mobile learning. Segundo a compilação de definições para aprendizagem móvel realizada por [Wu et al. 2012], pode-se dizer que na aprendizagem móvel, os estudantes realizam atividades educacionais, usando a tecnologia como ferramenta de mediação para aprendizagem via dispositivos móveis, acessando dados e se comunicando através da rede sem fio.

Nesse cenário, surge a tecnologia de podcast, que segundo [Deal 2007] é um meio de publicar arquivos de áudio ou vídeo na Web como uma série de episódios com um tema comum. Esses episódios são acompanhados por um arquivo de feed, que permite aos ouvintes subscreverem-se à uma série e receberem novos episódios automaticamente. [Deal 2007] destaca que o que difere um podcast de outros arquivos de áudio/vídeo na 
V Congresso Brasileiro de Informática na Educação (CBIE 2016)

Anais dos Workshops do V Congresso Brasileiro de Informática na Educação (CBIE 2016)

Web é o feed e o modelo de subscrição para entrega dos arquivos. Os podcasts, com a facilidade dos dispositivos móveis, podem ser ouvidos a qualquer hora e em qualquer lugar, sendo uma tecnologia com potencial para ser empregada para a educação.

Em relação ao uso educacional de podcasts, [Deal 2007] apresenta três usos mais comuns: a produção e distribuição de aulas para revisão, a entrega de material suplementar e a produção de podcasts pelos próprios estudantes, como parte de atividades avaliativas. [Deal 2007] argumenta que a melhoria na aprendizagem com o uso de podcasts depende do contexto educacional e das atividades de aprendizagem propostas.

Considerando as possibilidades educacionais dos podcasts na educação, em especial apoiando aprendizagem de conteúdos específicos enriquecido por tecnologias móveis, neste artigo propõe-se discutir o uso de podcasts na educação superior, como material suplementar para aprendizagem de algoritmos. O tema algoritmos faz parte de disciplinas iniciais dos cursos de graduação em Computação, sendo adequado para proposição deste trabalho.

Este artigo está organizado da seguinte forma. Na seção 2 são apresentados os podcasts, contemplando a sua utilização no panorama nacional. Na seção 3 são descritos trabalhos relacionados que utilizam podcasts na educação. Na seção 4, a série Algoritmo Podcast é detalhada. A percepção dos estudantes a respeito da série produzida é relatada na seção 5. Por fim, na seção 6 são descritas as considerações finais.

\section{Tecnologia podcast}

Podcast é uma forma de publicar conteúdo de áudio e vídeo na Internet como uma série de episódios com um tema em comum. Segundo [Deal 2007], algumas pessoas usam o termo podcast para se referir a qualquer distribuição de conteúdo de áudio ou vídeo na Web, mas tecnicamente falando, o feed e o modelo de subscrição de arquivo de entrega é o que diferencia um podcast de um outro arquivo na Web. Um agregador de podcast é um software que verifica os feeds de podcast em intervalos específicos, os atualiza e faz o download automático de quaisquer episódios novos desde a última verificação.

Uma pesquisa realizada no Brasil em 2014, denominada PodPesquisa ${ }^{1}$, revela a audiência de podcasts no país. A pesquisa ouviu 16 mil brasileiros, em todo o território nacional, dos quais $87.31 \%$ são homens e $12.69 \%$ são mulheres. A idade média dos ouvintes é de 25 anos. Do total de participantes, 54.7\% concluíram o ensino superior, seguidos de $18.15 \%$ com ensino médio.

Em relação ao dispositivo utilizado para ouvir os podcasts, os participantes declararam usar: computador (42.81\%), smartphone (42.33\%), iPod e semelhantes $(12.17 \%)$ e som automotivo (2.69\%). Sobre o local em que ouvem os podcasts, os participantes responderam: em casa $(45.93 \%)$, nos deslocamentos, carro ou transporte público (34.47\%), no trabalho (18.86\%) e na escola/faculdade $(0.74 \%)$.

Em relação a região do país, os ouvintes estão distribuídos da seguinte forma: Sudeste (57.10\%), Sul (19.10\%), Nordeste (12.20\%), Centro-Oeste (7.05\%), Norte $(2.59 \%)$ e Exterior (1.96\%).

Sobre o conteúdo ouvido, estão nas primeiras colocações: humor e entretenimento

\footnotetext{
${ }^{1}$ PodPesquisa 2014, disponível em http://podpesquisa.com.br/2014/resultado
} 
(86.30\%), televisão, séries e cinema (68.66\%), jogos (62.42\%), tecnologia (59.44\%), entre outros, destinando para educação $(19.03 \%)$.

Os dados da pesquisa permitem algumas reflexões. O público que ouve podcasts é um público jovem, predominantemente masculino, com alto nível de escolaridade, nas regiões mais desenvolvidas do país. Os podcasts são ouvidos tanto em computadores como em dispositivos móveis e os temas principais são ligados ao entretenimento. O uso da tecnologia podcasts não precisa ficar restrita a uma minoria elitizada, podendo alcançar todo o público, de Norte a Sul do país, incluindo mulheres e abrangendo outros temas, além do entretenimento.

Nesse contexto, almejando o acesso universal à informação com conteúdos de qualidade e aproveitando da tecnologia móvel, cada vez mais disponível para a população, propomos o desenvolvimento de uma série de podcasts educacionais, para ser utilizada por estudantes do ensino superior. A faixa etária, bem como o nível de escolaridade, dos estudantes de ensino superior está próxima do público ouvinte de podcasts. Os estudantes universitários podem fazer uso dos dispositivos móveis durante seu trajeto para universidade para ouvir podcasts educacionais sobre conteúdos relacionados aos seus cursos.

\section{Trabalhos Relacionados}

[Evans 2008] apresentou um estudo sobre o uso de podcast por estudantes do ensino superior. No estudo os alunos utilizaram podcasts com conteúdos de revisão de uma disciplina e foram questionados sobre seus benefícios. Os estudantes perceberam os podcasts como ferramentas mais eficientes para revisão de conteúdos do que livros textos. Além disso, os estudantes relataram que são mais receptivos ao material didático na forma de podcast do que as aulas tradicionais ou livros textos. Nesse contexto, o uso de podcast para adultos em ensino superior aparece como ferramenta promissora.

[Cunha et al. 2009] descrevem a utilização de podcast na graduação, em que propuseram aos estudantes transpor o material escrito de seus trabalhos para a forma de podcast de áudio ou vídeo. Com base na análise das experiências e opiniões dos alunos foi concluído que apesar das avaliações positivas, o uso de podcast não sobrepõe o formato de texto escrito. Diversos alunos relataram que a informação apresentada na forma de texto é assimilada mais facilmente, contudo a maioria dos estudantes se mostrou receptiva aos podcasts de áudio como complementos dos conteúdos escritos. Desse modo, mostra-se promissor o uso de podcasts complementando os materias tradicionais.

[Kurtz et al. 2007] utilizaram podcasts em vídeo com estudantes da disciplina de Engenharia de Software de um curso de graduação em Ciência da Computação. Os autores produziram podcasts correspondentes ao conteúdo da disciplina e trabalharam na forma de sala de aula invertida, ou seja, os estudantes assistiam os podcasts antes das aulas e resolviam problemas durante as aulas. Essa abordagem permitiu que o tempo de aula fosse dedicado a sessões de resolução de problemas e projetos. Analisando o aproveitamento, detectou-se que os estudantes que receberam aulas em podcast produziram projetos melhores do que a turma anterior da disciplina, a qual teve aulas convencionais.

Segundo [McGarr 2009], o uso de podcast no ensino superior resume-se a três modalidades: a mais comum é a de substituição, em que se usam gravações de aulas 
passadas com o propósito de revisão de conteúdos; a segunda mais encontrada é o uso suplementar, como material adicional; a terceira, e menos comum, é o uso criativo, em que os próprios estudantes produzem seus podcasts. $\mathrm{O}$ autor descreve que na substituição os estudantes tornam-se receptores passivos da informação, enquanto que no suplementar, os estudantes podem se aprofundar nos conteúdos e explorar novos pontos de vista. E por fim, o uso criativo é o que permite que os estudantes se tornem criadores de conhecimento ao invés de meros receptores. Por fim, [McGarr 2009] conclui que o uso de podcast e sua influência nas aulas tradicionais não deve ser determinada pela tecnologia em si, mas pela forma que é percebida pela instituição, seus docentes e alunos, sendo fortemente influenciado pela pedagogia dominante empregada.

Nesse contexto, o uso de podcasts no ensino superior, na forma de uso suplementar, mostra-se uma possibilidade educacional a ser explorada. No entanto, não foram encontrados trabalhos que relatem o uso de podcasts para ensino de Algoritmos, sendo este o objetivo deste trabalho.

\section{Série Algoritmo Podcast}

A série Algoritmo Podcast foi desenvolvida com o objetivo de servir como material suplementar para disciplinas de introdução a Algoritmos de cursos de graduação em Computação. O uso de podcast como material suplementar é relatado por [McGarr 2009], que ressalta que este uso possibilita que estudantes aprofundem-se nos conteúdos e explorem novos pontos de vista. Para o conteúdo de Algoritmos estabeleceu-se que os podcasts apresentariam os conteúdos com os conceitos iniciais, fornecendo exemplos do cotidiano dos estudantes.

O desenvolvimento da série Algoritmo Podcast envolveu 3 professores das disciplinas básicas de Algoritmos e Programação, que elaboraram os roteiros para gravação, bem como de 4 acadêmicos que realizaram as gravações, edições e distribuição dos áudios.

A metodologia de desenvolvimento dos episódios de podcast adotou um processo composto de três fases subsequentes, cada qual envolvendo atividades específicas [Deal 2007]. As fases do processo foram: a) produção de podcast; b) publicação de podcast; c) entrega e reprodução.

Os roteiros foram preparados priorizando uma linguagem informal e os assuntos foram abordados de maneira introdutória, respeitando a duração dos episódios, que ficou em torno de 5 minutos. A escolha do tempo de duração dos episódios está baseada nas conclusões de [Deal 2007], que relata uma pesquisa entre estudantes, apontando que a duração razoável para um podcast seria entre 6 e 10 minutos.

A série produzida é composta de 10 episódios que contemplam os assuntos:

1. Conceito de algoritmos

2. Tipos de dados e Operadores

3. Variáveis, constantes e estrutura sequencial

4. Estrutura condicional - se, se-senão

5. Estruturas de repetição - para..faça

6. Estruturas de repetição - enquanto..faça

7. Estruturas de repetição - faça..enquanto 
V Congresso Brasileiro de Informática na Educação (CBIE 2016)

Anais dos Workshops do V Congresso Brasileiro de Informática na Educação (CBIE 2016)

\section{Vetores}

9. Matrizes

10. Procedimentos e funções

Após a sua produção, a série Algoritmo Podcast foi disponibilizada através de um site $^{2}$ que pode ser visualizado na Figura 1. Pelo site é tanto possível ouvir diretamente os episódios, como fazer o download do arquivo.

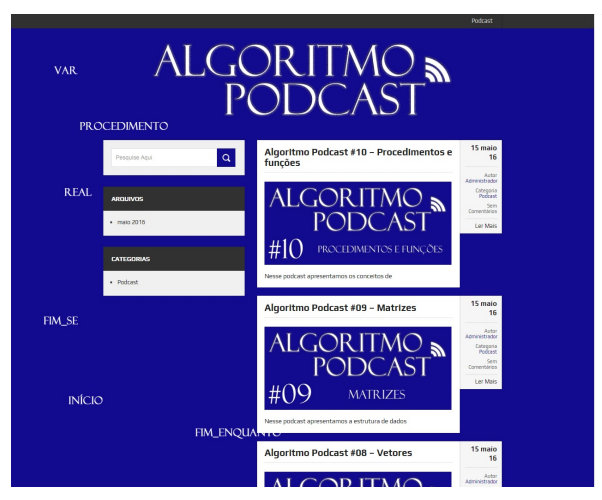

Figure 1. Site Algoritmo podcast.

Considerando a importância de ampla divulgação da série e do acesso através de dispositivos móveis, a mesma foi submetida a vários agregadores. Os agregadores podem ser instalados nos dispositivos móveis, e variam de acordo com o sistema operacional do aparelho. Para sistemas operacionais Android, os agregadores disponíveis são BeyondPod, Pocket Casts, Podcast Addict, Podcast Picker e Podcast Republic. Os agregadores para o sistema operacional iOS são Pocket Casts e Podcasts (Apple). E os agregadores para o sistema operacional Windows Phone são Pocket Casts e Podcast Picker.

Após finalizar as etapas de produção, publicação e entrega da série Algoritmo Podcast, a mesma foi indicada para graduandos cursando uma disciplina introdutória de Algoritmos.

\section{Uso educacional de podcasts}

A série foi apresentada durante um semestre letivo para uma turma da disciplina de Algoritmos de um curso de Sistemas de Informação. Durante o semestre, os estudantes responderam um questionário online sobre sua percepção a respeito dos podcasts.

Participaram da pesquisa 49 estudantes, com idade média de 22 anos, dos quais $10 \%$ são mulheres e $90 \%$ são homens. Se compararmos o perfil desses estudantes com a pesquisa sobre ouvintes de podcasts no Brasil [Sales Filho et al. 2014], veremos que os perfis não estão distantes, uma vez que nacionalmente temos idade média de 25 anos e $87.31 \%$ de homens e $12.69 \%$ de mulheres.

Perguntados a respeito do conhecimento prévio sobre podcasts: $18.4 \%$ dos alunos responderam que já conheciam essa tecnologia antes, $49 \%$ responderam que conheciam mais ou menos e o restante, $32.7 \%$ responderam que não sabiam o que era um podcast. Estes dados indicam que, mesmo sendo alunos de graduação em curso de Computação, os

\footnotetext{
${ }^{2}$ Série Algoritmo Podcast, Disponível em: www.algoritmopodcast.16mb.com
} 
V Congresso Brasileiro de Informática na Educação (CBIE 2016)

Anais dos Workshops do V Congresso Brasileiro de Informática na Educação (CBIE 2016)

estudantes não estão familiarizados com a tecnologia podcast. Considerando que os estudantes em questão estão localizados na região Centro-Oeste do país, estes dados também se relacionam com a pesquisa sobre uso de podcast no Brasil [Sales Filho et al. 2014], que relata que apenas $7.05 \%$ dos ouvintes estão localizados nessa região.

Os estudantes foram perguntados sobre o dispositivo em que ouviram os episódios da série: $36.6 \%$ usaram seus smartphones, $48.8 \%$ usaram seus computadores e/ou notebooks, enquanto $14.6 \%$ usaram ambos os dispositivos. Esses dados podem ser vistos na Figura 2. Comparando com a pesquisa nacional sobre o uso de podcast, o smartphone tem mais uso nacional, com $42.33 \%$ enquanto o computador tem menor uso nacional, com $42.81 \%$.

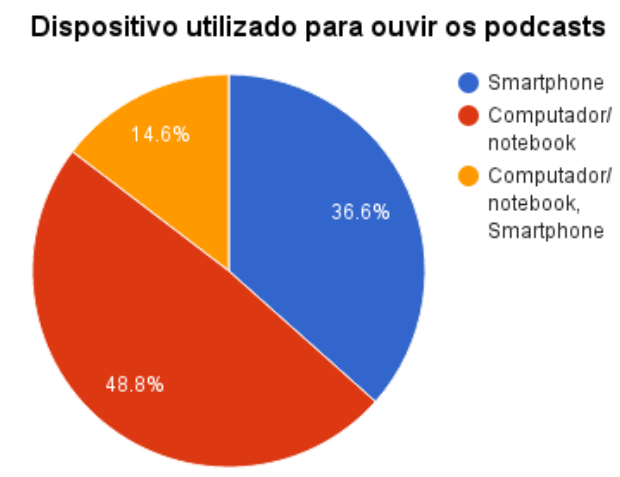

Figure 2. Dispositivos utilizados para ouvir a série Algoritmo Podcast.

Ainda, os estudantes foram perguntados sobre o melhor horário para ouvir os episódios da série Algoritmos Podcast: $54.3 \%$ dos alunos declararam que os escutaram em casa ou no trabalho, quando estavam estudando conteúdo da disciplina, outros $37 \%$ prefereriram escutar antes da aula, no trajeto para a universidade, enquanto $4.3 \%$ optaram por ouvir depois da aula ou antes da prova. Essas informações podem ser visualizadas na Figura 3. Nota-se que os alunos estão utilizando a mobilidade oferecida pelos dispositivos móveis para auxiliar seu aprendizado, uma vez que $37 \%$ deles preferiu ouvir os podcast durante seu trajeto para a universidade.

Para os estudantes que utilizaram seus smartphones para ouvir a série Algoritmos Podcast foi perguntado sobre quais agregadores utilizaram. Para Android a utilização foi: $23.1 \%$ Pocket Casts, $15.4 \%$ Podcast Republic, $15.4 \%$ BeyondPod e $7.7 \%$ Podcast Addict. Para o iphone, a utilização foi: $15.4 \%$ Podcasts (Apple) e 15.4\% Pocket Casts. Para o Windows Phone a utilização foi de $7.7 \%$ para o Pocket Casts. Essas informações podem ser vistas na Figura 4. Nota-se que o sistema android sobressai sobre os demais com $61.6 \%$ dos ouvintes, seguido do iOS com $38.5 \%$ e por último o Windows phone com $7.7 \%$.

Por fim, os estudantes foram perguntados sobre a utilidade da série Algoritmo Podcast para aprendizagem de algoritmos: $48.8 \%$ responderam concordo e $39 \%$ concordo totalmente, enquanto $7.3 \%$ responderam indiferente e $2.4 \%$ responderam tanto discordo 
V Congresso Brasileiro de Informática na Educação (CBIE 2016)

Anais dos Workshops do V Congresso Brasileiro de Informática na Educação (CBIE 2016)

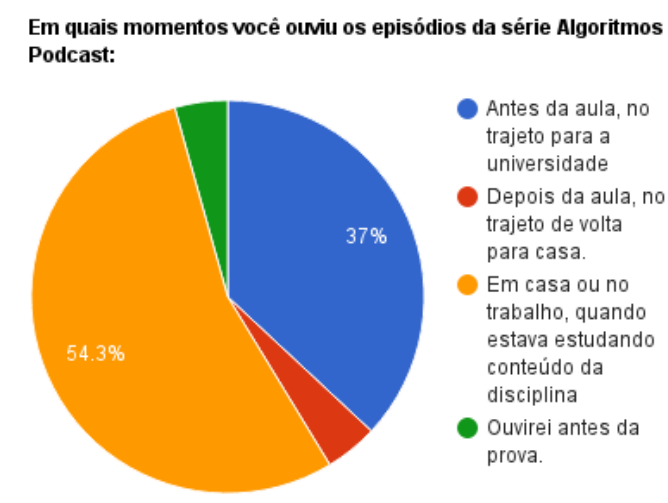

Figure 3. Momento em que estudantes ouviram a série Algoritmo Podcast.

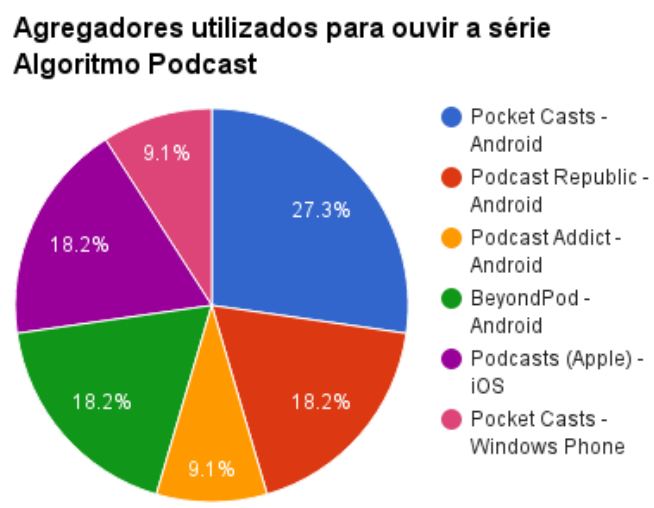

Figure 4. Agregadores utilizados para acessar a série Algoritmo Podcast.

como discordo totalmente. Esse resultado é animador, uma vez que entre concordo e concordo totalmente, tem-se $87.8 \%$ dos estudantes, o que significa que a maioria deles percebe a utilidade dos podcast para a aprendizagem. A utilidade dos podcasts pode ser vizualizada na Figura 5.

A utilização da série Algoritmo Podcast por estudantes de graduação mostra que a aprendizagem mediada por dispositivos móveis é possível, sendo percebida positivamente pelos estudantes. No início do semestre $32.7 \%$ dos estudantes declararam que não sabiam o que era um podcast e no final do semestre, $87.8 \%$ deles reconheceram sua utilidade para aprendizagem de algoritmos. Esses dados revelam que a utilização de tecnologia móvel na educação na forma de podcasts é um caminho promissor, devendo ser melhor explorado por educadores e estudantes.

\section{Considerações Finais}

A tecnologia podcast faz parte do dia-a-dia de muitos jovens, que utilizam as facilidades dos dispositivos móveis para ouvir podcasts em qualquer horário e local, inclusive du- 
V Congresso Brasileiro de Informática na Educação (CBIE 2016)

Anais dos Workshops do V Congresso Brasileiro de Informática na Educação (CBIE 2016)

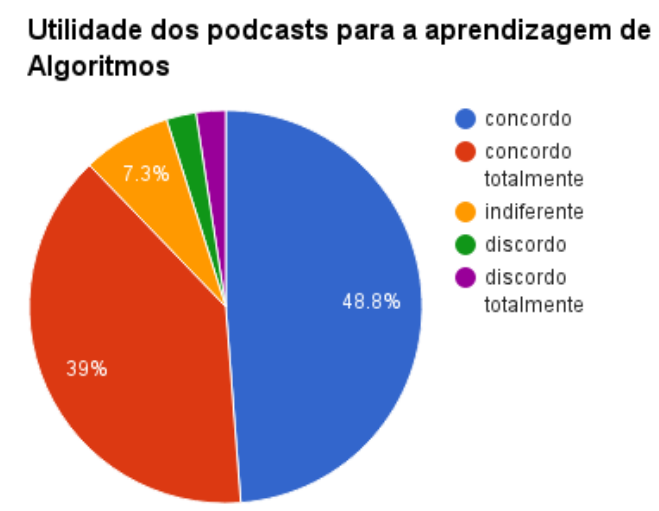

Figure 5. Percepção dos estudantes em relação a utilidade da Algoritmo podcast para aprendizagem.

rante seus deslocamentos [Sales Filho et al. 2014]. Para podermos incorporar as vantagens de uso de podcasts na aprendizagem com dispositivos móveis, é necessário que séries educacionais estejam disponíveis. Nesse contexto, o presente trabalho contribui para a disponibilização da série Algoritmo Podcast, que contempla o conteúdo de disciplinas introdutórias de algoritmos.

A utilização de dispositivos móveis na educação pode contribuir para melhoria na aprendizagem, sendo um campo amplo ainda a ser explorado. A tecnologia podcast, já em uso por muitos jovens, é mais uma forma de aproximar os estudantes do conteúdo, de uma forma agradável, agregando as facilidades de a qualquer tempo e em qualquer lugar.

Como trabalhos futuros, pretende-se elaborar novas séries, aumentando os conteúdos disponíveis nessa tecnologia para os estudantes. Além disso, é necessário realizar pesquisas sobre o impacto dos podcasts no desempenho dos estudantes após ouvir as séries. No entanto, tomando como base os trabalhos relacionados, espera-se bons resultados.

\section{References}

Cunha, C., Teixeira, S., Maciel, R., and Aguiar, C. (2009). Aprender com podcasts. In Actas do X Congresso Internacional Galego-Português de Psicopedagogia. Braga: Universidade do Minho, pages 5493-5498.

Deal, A. (2007). Podcasting. Teaching with Technology.

Evans, C. (2008). The effectiveness of m-learning in the form of podcast revision lectures in higher education. Computers \& education, 50(2):491-498.

Kurtz, B. L., Fenwick Jr, J. B., and Ellsworth, C. C. (2007). Using podcasts and tablet pcs in computer science. In Proceedings of the 45th annual southeast regional conference, pages 484-489. ACM.

McGarr, O. (2009). A review of podcasting in higher education: Its influence on the traditional lecture. Australasian Journal of Educational Technology, 25(3). 
V Congresso Brasileiro de Informática na Educação (CBIE 2016)

Anais dos Workshops do V Congresso Brasileiro de Informática na Educação (CBIE 2016)

Sales Filho, E., Toledano, G., Lopes, L., and Ferreira, R. (2014). Podpesquisa. Disponível em: http://podpesquisa.com.br/2014/resultado.

Wu, W.-H., Wu, Y.-C. J., Chen, C.-Y., Kao, H.-Y., Lin, C.-H., and Huang, S.-H. (2012). Review of trends from mobile learning studies: A meta-analysis. Computers \& Education, 59(2):817-827. 